\title{
Review Article \\ Prophylactic Mesh Application during Colostomy to Prevent Parastomal Hernia: A Meta-Analysis
}

\author{
JunJia Zhu, YuWei Pu, XiaoDong Yang, DeBao Zhang, Kui Zhao, \\ Wei Peng, and ChunGen Xing \\ Department of General Surgery, Second Affiliated Hospital, Soochow University, Suzhou, Jiangsu 215004, China \\ Correspondence should be addressed to ChunGen Xing; daoketezjj@126.com
}

Received 26 June 2016; Accepted 28 August 2016

Academic Editor: Werner A. Draaisma

Copyright (c) 2016 JunJia Zhu et al. This is an open access article distributed under the Creative Commons Attribution License, which permits unrestricted use, distribution, and reproduction in any medium, provided the original work is properly cited.

\begin{abstract}
Background. Parastomal hernia is a common complication after stoma formation, especially in permanent colostomy. The present meta-analysis aimed to evaluate the effectiveness of prophylactic mesh application during permanent colostomy for preventing parastomal hernia. Methods. Randomized controlled trials comparing outcomes in patients who underwent colostomy with or without prophylactic mesh application were identified from PubMed, EMBASE, Science Citation Index, and the Cochrane Libraries. Results. This meta-analysis included 8 randomized controlled trials with 522 participants. Our pooled results showed that prophylactic mesh application (mesh group) reduced the incidence of clinically detected parastomal hernia (risk ratio [RR]: 0.22; $95 \%$ confidence interval [CI]: $0.13-0.38 ; P<0.00001$ ), radiologically detected parastomal hernia (RR: 0.62 ; $95 \%$ CI: $0.47-$ $0.82 ; P=0.0008$ ), and surgical repair for herniation (RR: $0.34 ; 95 \% \mathrm{CI}: 0.14-0.83 ; P=0.02$ ) when compared with conventional permanent colostomy formation (control group). The incidence of complications, including wound infection, peristomal infection, mesh infection, stomal necrosis and stenosis, stoma site pain, and fistula, was not higher in the mesh group than in the control group. Conclusions. Our meta-analysis demonstrated that prophylactic mesh application at the time of primary colostomy formation is a promising method for the prevention of parastomal herniation.
\end{abstract}

\section{Introduction}

Parastomal hernia is one of the most frequent colorectal complications noted in patients who have undergone surgical treatment for cancer or inflammatory bowel disease, followed by stoma formation [1]. It is known that colostomy often has an adverse influence on quality of life [2]. The prevalence of parastomal hernia ranges from $5 \%$ to $52 \%$ in the literature [35], and the rate of colostomy-related parastomal hernia has been found to gradually increase with prolonged follow-up time $[4,6]$.

Although most parastomal hernias remain asymptomatic, many patients complain of pain, stomal appliance problems, skin irritation, and stoma site discomfort [7, 8]. Surgical techniques for repairing parastomal hernias include local fixation, resiting the stoma, and prosthetic mesh repair [9]. However, the recurrence rate remains high after surgical treatment of parastomal hernia [10]. According to a report by Allen-Mersh and Thomson, $47 \%$ of patients who have undergone local repair experience hernia recurrence [11]. Prosthetic mesh repair has been recommended by Carne et al., as this approach is associated with low recurrence; however, this technique requires a second surgery and can cause additional complications [12].

Satisfactory techniques for repairing parastomal hernia are lacking, and, therefore, prophylaxis of parastomal hernia is extremely important $[12,13]$. Lian et al. reported a low rate of parastomal hernia with extraperitoneal colostomy [14]. Presently, more surgeons are considering prophylactic mesh application at the time of stoma creation. Bayer et al. first described this procedure in 1986 and reported that no parastomal hernia formation was detected in 47 patients [15]. Several previous studies have reported a low rate of hernia formation and a reduced risk of infection with prophylactic 
mesh application [16-18]. In a previous study by Fleshman et al., there was no difference in the incidence of parastomal herniation between the mesh group and conventional group [19]. However, ileostomy accounted for a large proportion of the procedures along with open and laparoscopic surgery, and maybe this affected the statistical power to detect the differences between two groups. In a report by Carne et al., $4.0-48.1 \%$ of colostomies developed parastomal hernias [12]. Considering colostomy is more susceptible to developing the parastomal hernia than ileostomy, the present metaanalysis aimed to evaluate the effectiveness of prophylactic mesh application during permanent colostomy for preventing parastomal hernia.

\section{Materials and Methods}

2.1. Search Strategy and Selection Criteria. Multiple databases (PubMed, EMBASE, Science Citation Index, the Cochrane Central Register of Controlled Trials, and Cochrane Register of Systematic Reviews) were searched. The literature search was performed for studies published between January 1980 and April 2016, using the following medical subject headings: "surgical mesh," "implants," "enterostomy," "ostomy," "surgical stomas," and "colostomy," along with free-text words. Two reviewers scanned and evaluated the studies independently.

The inclusion criteria were as follows: (1) comparative trials comparing primary formation of the colostomy with or without prophylactic mesh application; (2) randomized controlled trials (RCTs) published before March 2016; and (3) presence of information on the following outcomes: parastomal hernia, surgery for parastomal hernia, and infectious and noninfectious complications.

2.2. Data Collection. Two investigators extracted and documented the relevant information from each study independently, and disagreements were resolved through consultations with a third investigator. If disagreements remained unresolved, the whole study group participated in discussions. The following data were extracted: author, country, year of publication, participant parameters, surgical parameters, sample size, diagnostic method for hernia, and followup time. The following parameters were extracted: occurrence of parastomal hernia, incidence of surgery repair for parastomal hernia, and stoma-related and non-stoma-related complications (wound infection, peristomal infection, mesh infection, fistula, stomal necrosis and stenosis, and stomal site pain).

2.3. Quality Assessment. We evaluated the methodological quality of the included studies according to the Cochrane Risk of Bias Tool [29]. The assessment included the following 7 items [30]: randomization sequence generation, allocation concealment, blinding of participants and study personnel, blinding of outcome assessors, incomplete outcome data, selective reporting, and other biases. Two investigators resolved disagreements through discussion.

2.4. Statistical Analysis. All analyses were performed using the Review Manager software ver. 5.3 (the Nordic Cochrane
Centre, Copenhagen, Denmark, 2014). The analyses were performed using risk ratios (RRs) and 95\% confidence intervals (CIs) for dichotomous data. A $P$ value $<0.05$ was considered statistically significant.

Heterogeneity was assessed with $I^{2}$ measurement across the studies [31]. Statistical heterogeneity was assessed with $I^{2}$ measurement and was regarded as significant when $I^{2}$ was $>50 \%$ and $P$ value was $\leq 0.10$. A random-effects model was used to combine the data if heterogeneity was present in the results; otherwise, a fixed-effect model was used.

We did not assess publication bias because a small number of trials were included in this meta-analysis.

\section{Results}

3.1. Included Studies and Study Characteristics. The search of the electronic databases identified 782 relevant studies. Of these studies, 171 duplicates were excluded. Additionally, 2 investigators screened the title and abstract independently and excluded 581 studies. Thus, 30 studies were finally considered for inclusion. Of these studies, 8 RCTs with 522 participants were finally selected for this meta-analysis $[18,20-$ 28]. The selection procedure has been presented in Figure 1.

The basic characteristics of the RCTs are presented in Table 1. The 522 participants enrolled in the RCTs were divided into the following 2 groups: mesh group (underwent permanent colostomy with prophylactic mesh application) and control group (underwent conventional colostomy). The follow-up time ranged from 3 to 60 months. Patients lost to follow-up were taken into account in all studies, except the trial by Vierimaa et al. [27]; therefore, per protocol analysis was applied at the end. The results of the methodological quality of the included studies are presented in Figure 2.

\subsection{Outcomes of the Pooled Studies}

3.2.1. Parastomal Hernia. All 8 RCTs reported the incidence of parastomal hernia. Three trials used clinical assessment $[18,20,21,24,25], 2$ trials used computed tomography (CT) to detect parastomal hernia $[23,28]$, and the remaining 3 trials used both methods [22, 26, 27]. Six trials showed that the rate of clinically detected parastomal hernia was lower in the mesh group than in the control group (RR: 0.22; 95\% CI: $0.13-$ $0.38 ; P<0.00001$ ) (Figure 3) [18, 20-22, 24-27]. Cingi et al. reported a higher rate of parastomal hernia when CT was used as the diagnostic tool than when clinical assessment was used [32]. Additionally, radiological method also favoured the prophylactic mesh as a positive technique in prevention of parastomal hernia (RR: 0.62 ; 95\% CI: $0.47-0.82 ; P=0.0008$ ) (Figure 4). Accordingly, the diagnostic rate was higher with CT than with clinical assessment.

Seven trials reported surgical repair for parastomal hernia [21-24, 26-28]. The surgical repair rate for parastomal hernia was lower in the mesh group than in the control group (RR: 0.34; 95\% CI: 0.14-0.83; $P=0.02$ ) (Figure 5).

3.2.2. Infectious Complications. Pooled data from the trials showed that applying a mesh at the time of fashioning the 


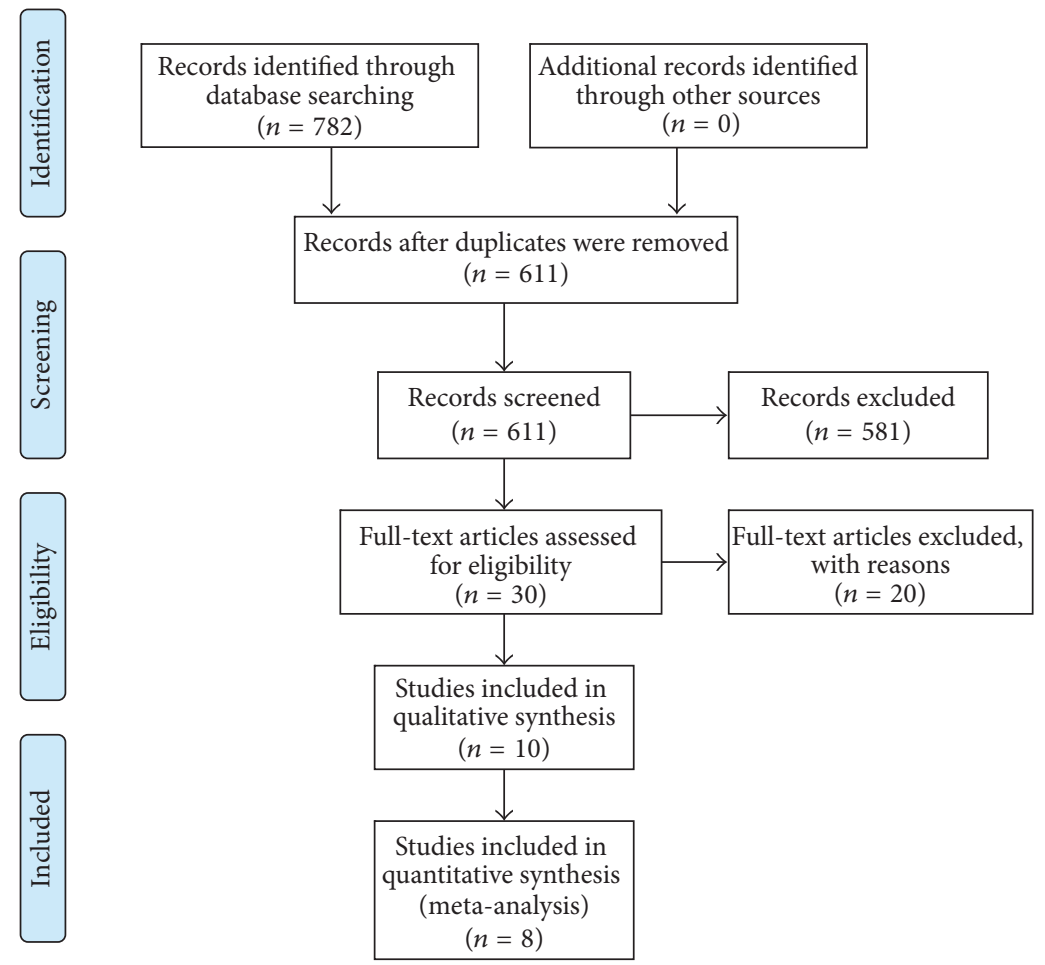

FIGURE 1: Flow chart of searching strategy for randomized controlled trials.

colostomy would not increase the risk of wound infection (RR: 0.74; 95\% CI: 0.33-1.64; $P=0.46$ ) (Figure 6) and peristomal infection (RR: $0.52 ; 95 \% \mathrm{CI}: 0.10-2.80 ; P=$ 0.45) (Figure 7). Additionally, according to 4 trials, mesh application did not increase the incidence of infectious complications related to the mesh $[21,23,25,28]$. The perineal wound infection rate was higher in the mesh group than in the control group; however, the difference was not significant (RR: 1.54; 95\% CI: 0.82-2.89; $P=0.17$ ) (Figure 8).

3.2.3. Noninfectious Complications. There were no differences in the incidences of stomal necrosis (RR: 0.58; 95\% CI: $0.22-$ $1.50 ; P=0.26$ ) (Figure 9) and stomal stenosis (RR: 1.67; 95\% CI: $0.36-7.75 ; P=0.51$ ) (Figure 10 ) between the mesh and control groups.

\section{Discussion}

Presently, patients who undergo colostomy seek a high quality of life. However, a large proportion of patients develop complications related to colostomy [33], and sometimes these complications can be life-threatening [34]. Among these complications, parastomal hernia is one of the major issues. One-third of patients who develop parastomal hernias may need operative treatment [35, 36]. Although techniques, such as mesh reinforcement and stoma repositioning, have been used to repair an affected abdominal wall, the results may be frustrating [12]. Therefore, the prevention of parastomal hernia is extremely important. Prophylactic mesh application at the time of stoma formation appears to help prevent herniation [37, 38]. Considering that parastomal hernia occurs frequently after colostomy [12, 39], our metaanalysis on the benefit of prophylactic mesh application after colostomy is important. Several systematic reviews have discussed whether prophylactic mesh application is beneficial [1, 40-42]; however, only 3 RCTs with small sample sizes were evaluated, both colostomy and ileostomy were considered, and mesh-related complications were not appropriately assessed. In our meta-analysis, 4 single-center and 4 multicenter studies with 522 participants were pooled to evaluate the value of the prophylactic mesh.

We found that, in clinical and radiological assessments, the occurrence of parastomal herniation reduced with prophylactic mesh application. Additionally, according to our pooled result, the operative treatment for parastomal hernia reduced with prophylactic mesh application. One trial in our meta-analysis reported that the stoma aperture was much smaller in the mesh group than in the control group, indicating that patients in the control group were susceptible to the development of parastomal hernia [26]. The follow-up time of the enrolled studies ranged from 3 to 60 months. Considering that the occurrence of parastomal hernia increases as the follow-up time is prolonged [43], a short follow-up time in the trial by Brandsma et al. might have caused a potential bias [25]. However, the occurrence of parastomal hernia remained low in the mesh group after elimination of this study (RR: 0.22; 95\% CI: $0.13-0.37 ; P<0.00001)$.

It appears that the use of CT may contribute to a high detection rate of parastomal hernia $[4,32]$, and this is 


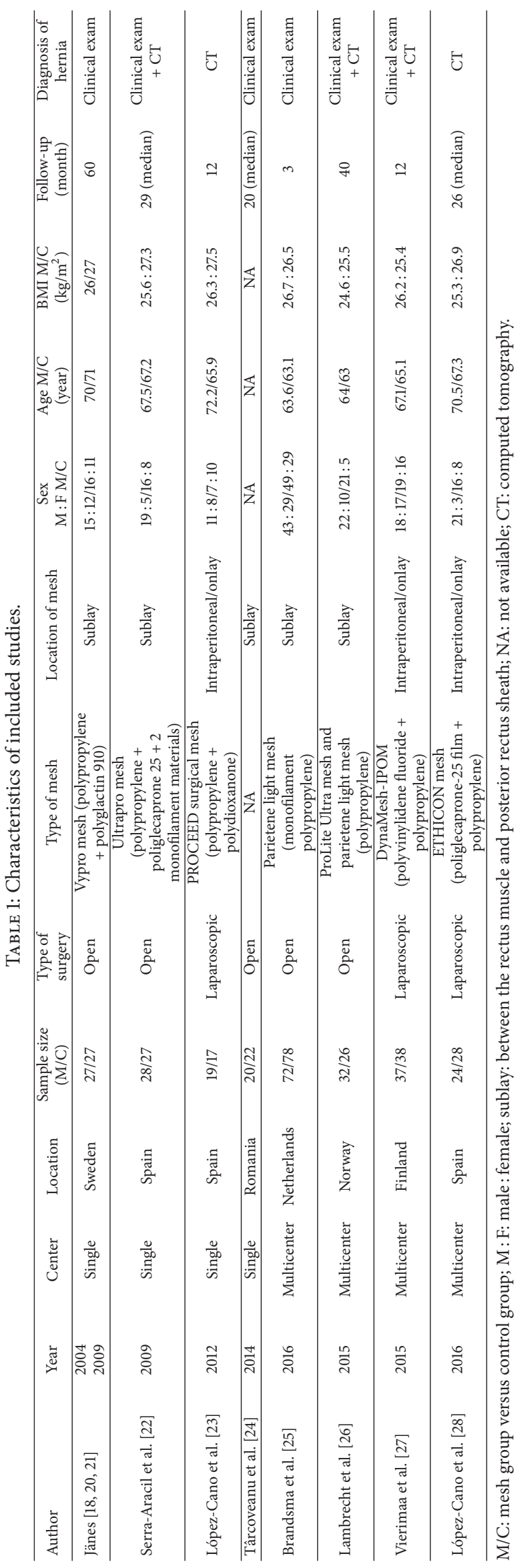




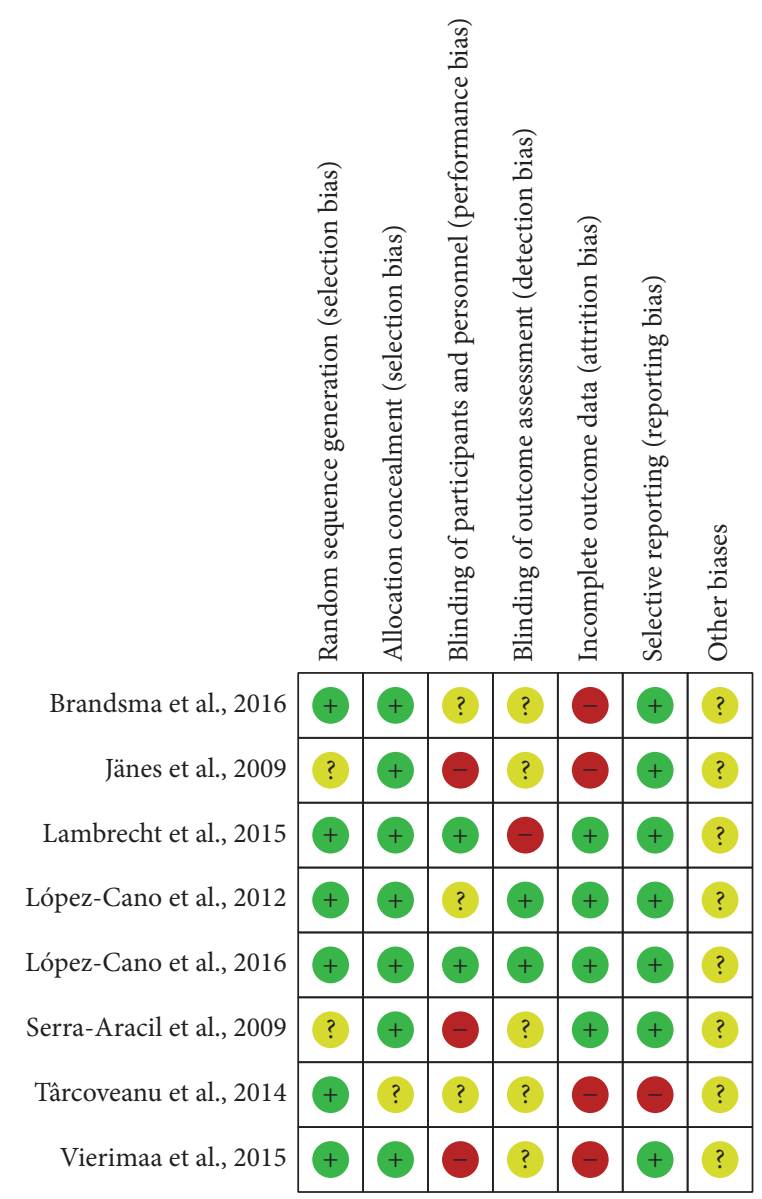

Figure 2: Risk of bias summary: author's judgement about risk of bias according to the Cochrane Risk of Bias Tool. +, high quality, -, low quality, and ?, unable to determine.

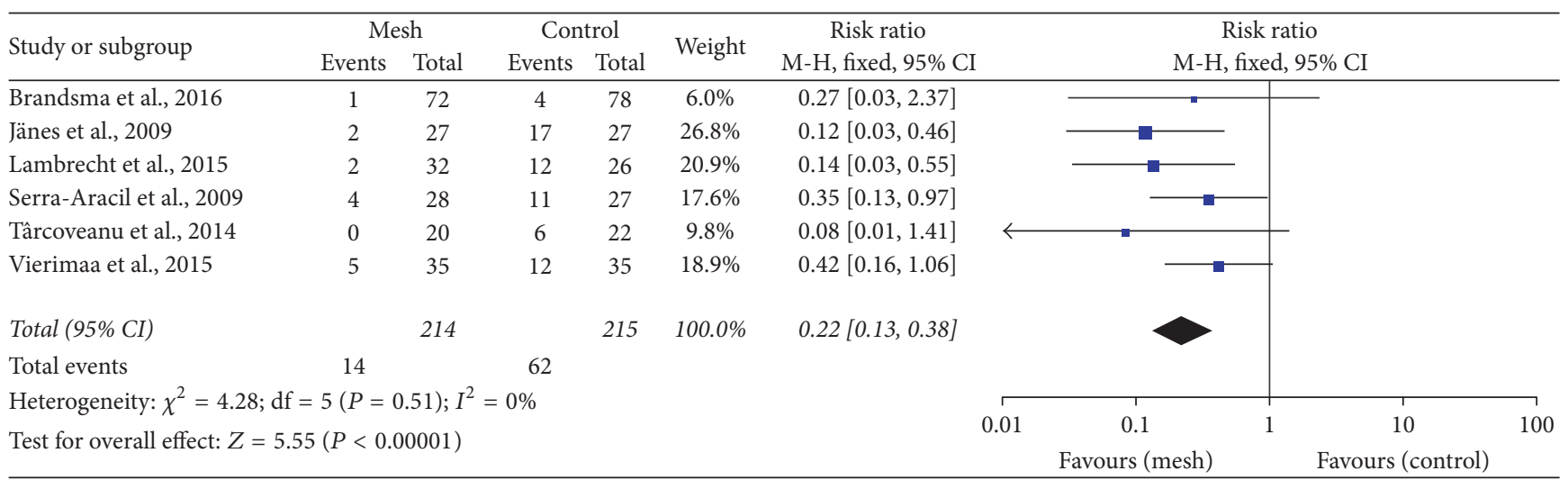

FIGURE 3: Forest plot for clinically detected parastomal hernia.

consistent with our result (Figures 3 and 4). Janson et al. reported that prophylactic mesh application could reduce the rate of parastomal hernia formation in patients undergoing laparoscopic sigmoidostomy [44]. However, as this previous study was not a controlled trial, it could not be deduced whether the laparoscopic technique was superior to the open technique. In our meta-analysis, patients underwent laparoscopic surgeries with the placement of a mesh in an intraperitoneal/onlay position $[23,27,28]$ and underwent open surgery in a sublay position $[18,21,22,24-26]$. Considering that most patients underwent clinical examinations to detect parastomal hernia in the open surgery group and underwent CT in the laparoscopy group, we did not assess the most appropriate type of surgery or position of the 


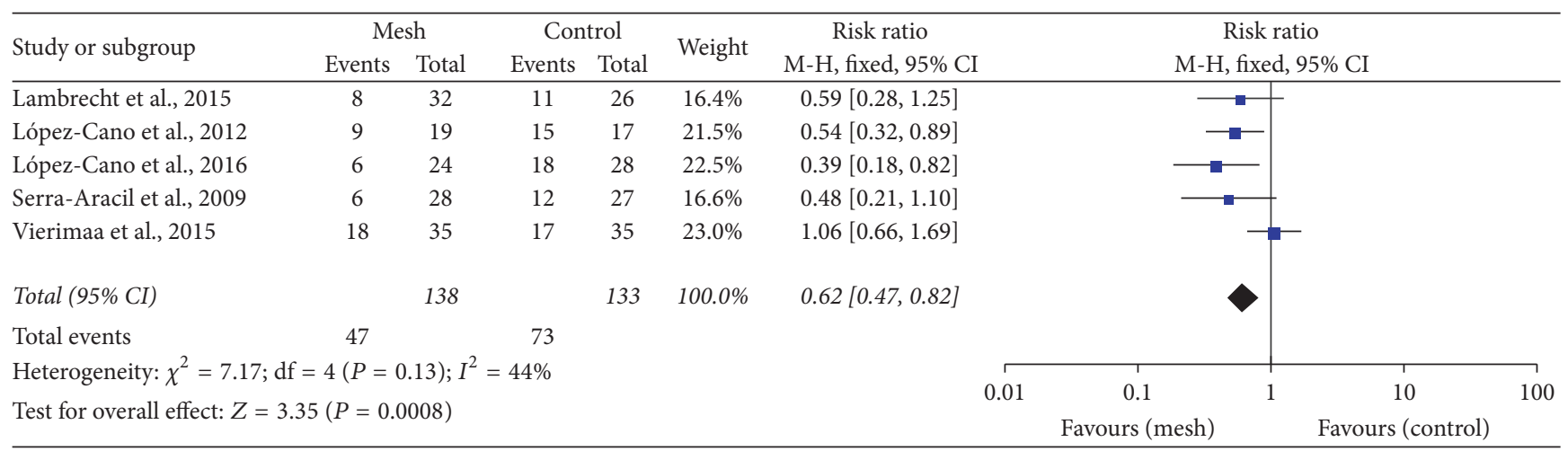

FIGURE 4: Forest plot for radiologically detected parastomal hernia.

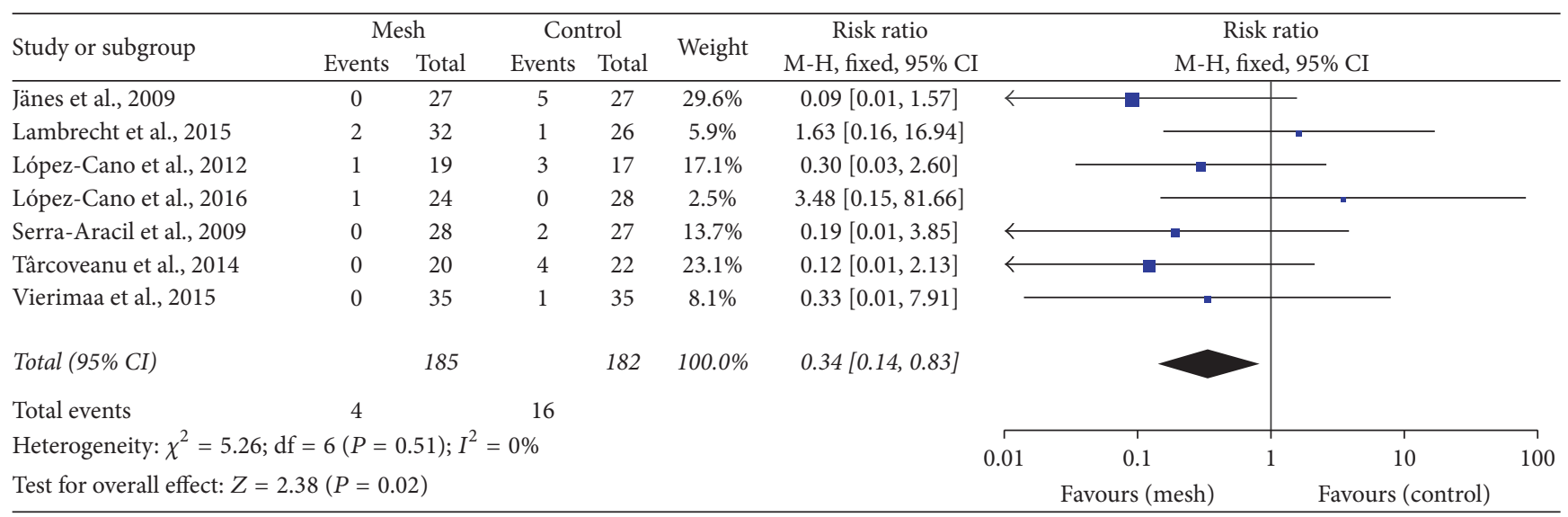

FIGURE 5: Forest plot for surgical repair for parastomal hernia.

mesh. Further trials are needed to compare different surgical procedures involving prophylactic mesh application.

Surgeons have been worried that mesh application close to the intestine may increase the risk of infection and cause complications, such as fistula and intestinal obstruction [45]. Our meta-analysis found that the rate of infection, including wound infection and peristomal infection, did not increase with mesh application (Figures 6 and 7). Four trials reported that no mesh-related infection occurred $[21,23,25,28]$. The perineal infection rate tended to be higher in the mesh group than in the control group (without significance), and this might be associated with the abdominoperineal resection itself rather than with the existence of the mesh [46]. In addition, we noted that stomal necrosis and stenosis were not higher in the mesh group than in the control group (Figures 9 and 10). In 4 trials, fistula formation was not noted in the mesh group $[21,22,24,26]$. Moreover, mesh application did not cause pain at the stomal site $[25,27]$. Our pooled results are consistent with the findings of some non-RCTs that showed promising outcomes with mesh placement at the time of colostomy formation and absence of an increase in the complication rate $[38,47]$. Lee et al. performed a cost effectiveness analysis and found that the cost was lower and the ability to prevent parastomal hernia was greater with prophylactic mesh application in patients who underwent permanent colostomy than with the conventional method [48].

In the included trials, surgeons used the keyhole technique, which involves an incision at the center of the mesh. The disadvantage of this approach is that the incision will enlarge after a long follow-up [23, 28]. Laparoscopy is developing rapidly and is being widely used; however, a new technique for mesh application is needed. A new method named Sugarbaker and its modifications have been widely applied in laparoscopy $[49,50]$. The recurrence rate after parastomal hernia repair was found to be lower with the Sugarbaker technique than with the keyhole technique [51]. In a trial by López-Cano et al., this new technique showed promising results with regard to the prevention of parastomal hernia $[28,52]$. Further RCTs should be performed to confirm the effectiveness of this new technique.

However, the present meta-analysis had some limitations. First, the sample size was not sufficiently large, and this might have resulted in bias. Second, we failed to evaluate the most optimal position, mesh material, and surgical type. Therefore, further trials with a larger sample size should be 


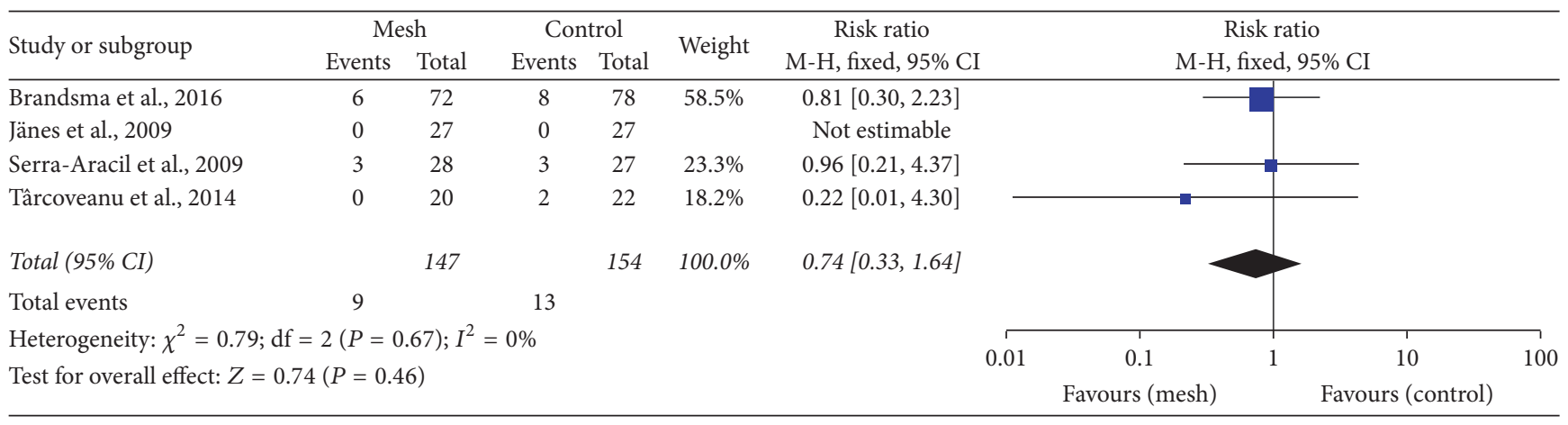

FIGURE 6: Forest plot for wound infection.

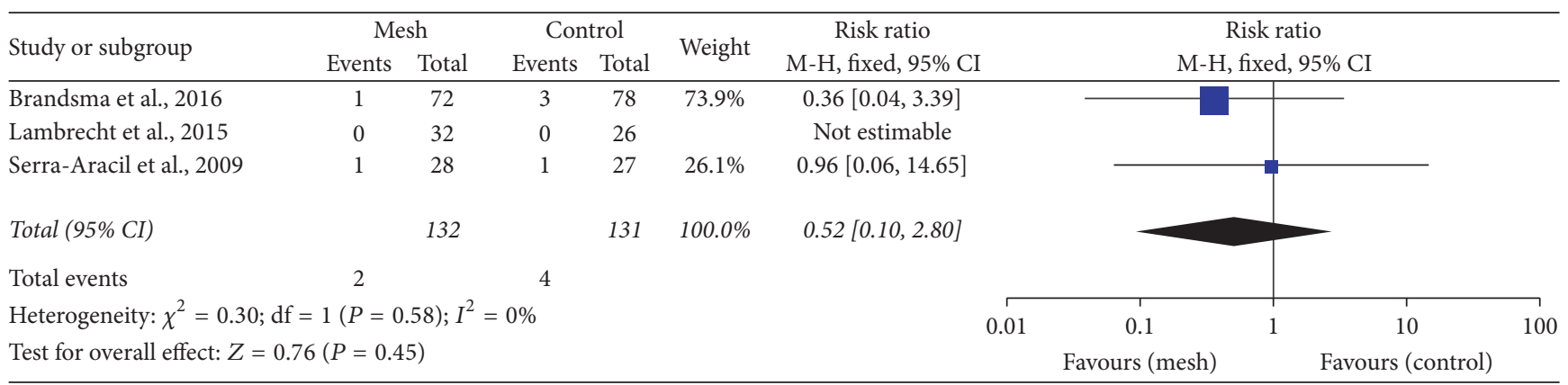

FIGURE 7: Forest plot for peristomal infection.

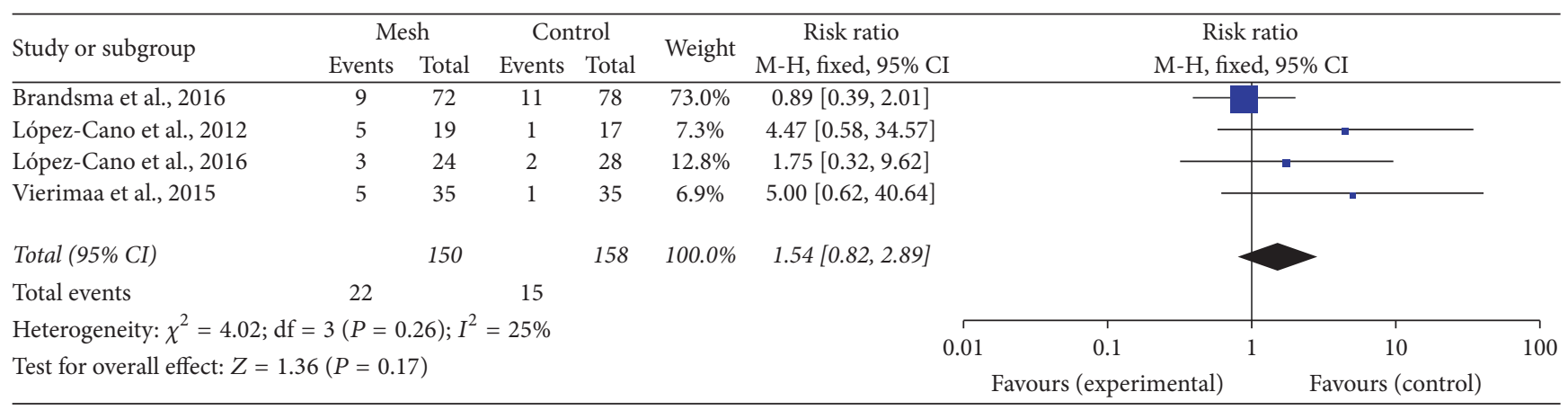

FIGURE 8: Forest plot for perineal wound infection.

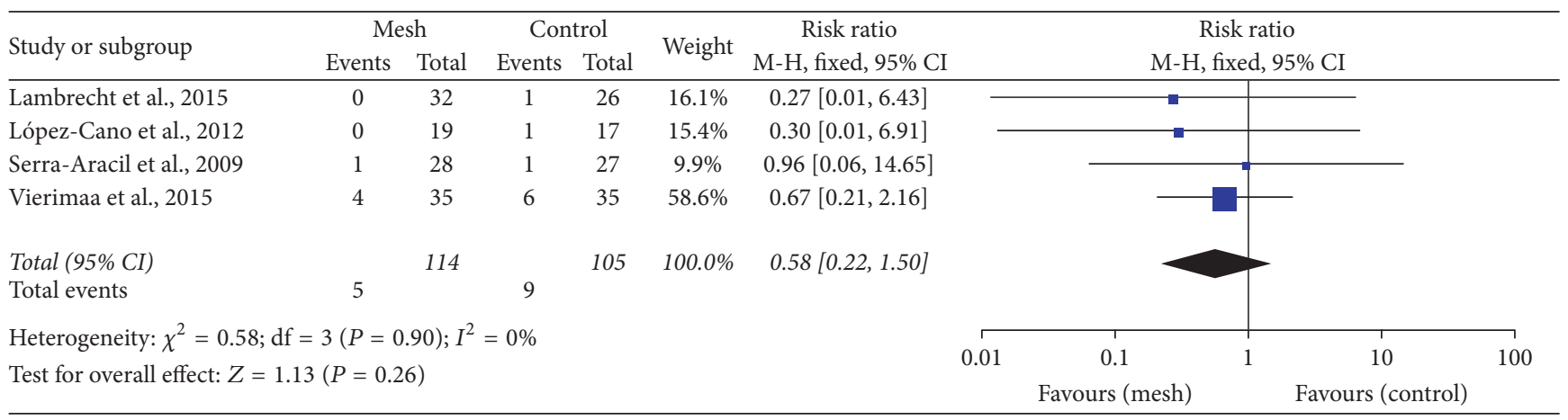

FIgURE 9: Forest plot for stomal necrosis. 


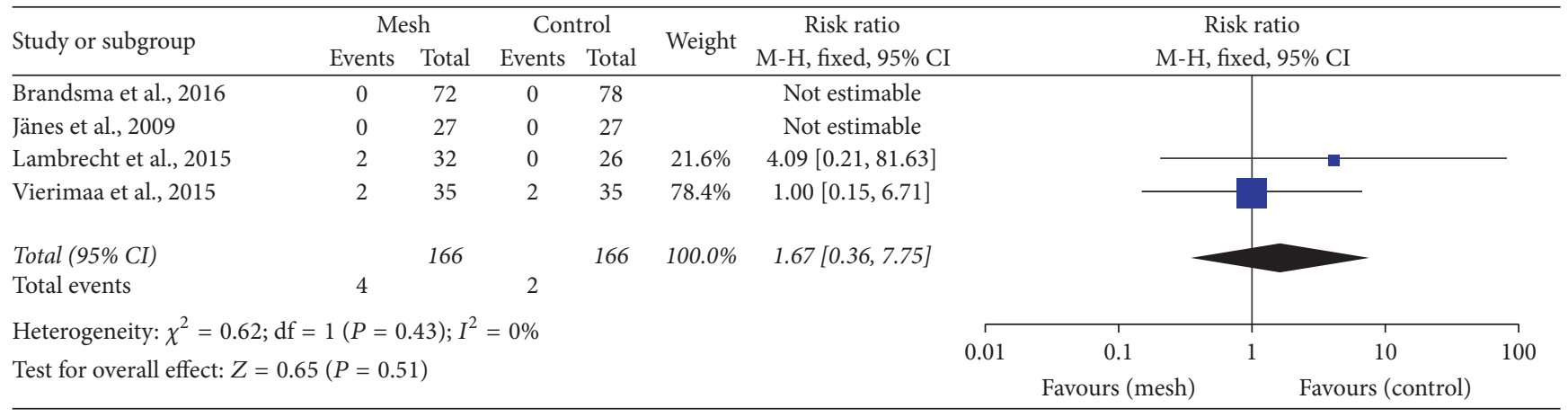

FIGURE 10: Forest plot for stomal stenosis.

performed to ensure a reliable and powerful analysis. Finally, we included some trials with a short follow-up. Considering that the occurrence of parastomal hernia increases after 10 years [53], trials with a longer follow-up period are needed to confirm the results.

\section{Conclusion}

Our meta-analysis demonstrated that prophylactic mesh application at the time of primary colostomy formation is a promising method for the prevention of parastomal herniation. This approach might not completely prevent parastomal herniation but might reduce the incidence of parastomal herniation without increasing the incidence of complications in the long term. Therefore, it may be the preferred option in patients undergoing permanent colostomy.

\section{Competing Interests}

There are no competing interests regarding the publication of this study.

\section{Authors' Contributions}

JunJia Zhu and YuWei Pu contributed equally to this work. JunJia Zhu and YuWei Pu helped conceive and design the study. JunJia Zhu, YuWei Pu, and DeBao Zhang performed the study. JunJia Zhu and Kui Zhao conducted the literature search. JunJia Zhu, YuWei Pu, and XiaoDong Yang collected and analyzed the data. JunJia Zhu and Wei Peng wrote the paper. JunJia Zhu and ChunGen Xing revised the manuscript.

\section{Acknowledgments}

This work was supported by the Innovation Fund Designated for Graduate Students of Jiangsu Province (SJLX15_0577), the National Natural Science Foundation of China (no. 81672970), the Second Affiliated Hospital of Soochow University preponderant clinic discipline group project funding (XKQ2015007), and Suzhou Key Medical Center (LCZX201505).

\section{References}

[1] M. S. Sajid, L. Kalra, K. Hutson, and P. Sains, "Parastomal hernia as a consequence of colorectal cancer resections can prophylactically be controlled by mesh insertion at the time of primary surgery: a literature based systematic review of published trials," Minerva Chirurgica, vol. 67, no. 4, pp. 289-296, 2012.

[2] C. T. Aquina, J. C. Iannuzzi, C. P. Probst et al., "Parastomal hernia: a growing problem with new solutions," Digestive Surgery, vol. 31, no. 4-5, pp. 366-376, 2014.

[3] J. T. Mäkelä, P. H. Turku, and S. T. Laitinen, "Analysis of late stomal complications following ostomy surgery," Annales Chirurgiae et Gynaecologiae, vol. 86, no. 4, pp. 305-310, 1997.

[4] L. A. Israelsson, "Parastomal hernias," The Surgical Clinics of North America, vol. 88, no. 1, pp. 113-125, 2008.

[5] M.-T. Cheung, N.-H. Chia, and W.-Y. Chiu, "Surgical treatment of parastomal hernia complicating sigmoid colostomies," Diseases of the Colon and Rectum, vol. 44, no. 2, pp. 266-270, 2001.

[6] E. Mylonakis, M. Scarpa, M. Barollo, C. Yarnoz, and M. R. B. Keighley, "Life table analysis of hernia following end colostomy construction," Colorectal Disease, vol. 3, no. 5, pp. 334-337, 2001.

[7] J. Hardt, J. J. Meerpohl, M.-I. Metzendorf, P. Kienle, S. Post, and F. Herrle, "Lateral pararectal versus transrectal stoma placement for prevention of parastomal herniation," The Cochrane Database of Systematic Reviews, no. 11, Article ID CD009487, 2013.

[8] J. Al Shakarchi and J. G. Williams, "Systematic review of open techniques for parastomal hernia repair," Techniques in Coloproctology, vol. 18, no. 5, pp. 427-432, 2014.

[9] N. Rieger, J. Moore, P. Hewett, S. Lee, and J. Stephens, "Parastomal hernia repair," Colorectal Disease, vol. 6, no. 3, pp. 203205, 2004.

[10] P. Wara, "Parastomal hernia repair. An update," Minerva Chirurgica, vol. 66, no. 2, pp. 123-128, 2011.

[11] T. G. Allen-Mersh and J. P. S. Thomson, "Surgical treatment of colostomy complications," The British Journal of Surgery, vol. 75, no. 5, pp. 416-418, 1988.

[12] P. W. G. Carne, G. M. Robertson, and F. A. Frizelle, "Parastomal hernia," The British Journal of Surgery, vol. 90, no. 7, pp. 784-793, 2003.

[13] L. Martin and G. Foster, "Parastomal hernia," Annals of the Royal College of Surgeons of England, vol. 78, no. 2, pp. 81-84, 1996.

[14] L. Lian, X.-R. Wu, X.-S. He et al., "Extraperitoneal vs. intraperitoneal route for permanent colostomy: a meta-analysis of 1,071 
patients," International Journal of Colorectal Disease, vol. 27, no. 1, pp. 59-64, 2012.

[15] I. Bayer, S. Kyzer, and C. Chaimoff, "A new approach to primary strengthening of colostomy with Marlex mesh to prevent paracolostomy hernia," Surgery Gynecology \& Obstetrics, vol. 163, no. 6, pp. 579-780, 1986.

[16] D. Berger, "Prevention of parastomal hernias by prophylactic use of a specially designed intraperitoneal onlay mesh (Dynamesh IPST ${ }^{\circledR}$ )," Hernia, vol. 12, no. 3, pp. 243-246, 2008.

[17] T. M. Hammond, A. Huang, K. Prosser, J. N. Frye, and N. S. Williams, "Parastomal hernia prevention using a novel collagen implant: a randomised controlled phase 1 study," Hernia, vol. 12, no. 5, pp. 475-481, 2008.

[18] A. Jänes, Y. Cengiz, and L. A. Israelsson, "Preventing parastomal hernia with a prosthetic mesh: A Randomized Study," Archives of Surgery, vol. 139, no. 12, pp. 1356-1358, 2004.

[19] J. W. Fleshman, D. E. Beck, N. Hyman, S. D. Wexner, J. Bauer, and V. George, "A prospective, multicenter, randomized, controlled study of non-cross-linked porcine acellular dermal matrix fascial sublay for parastomal reinforcement in patients undergoing surgery for permanent abdominal wall ostomies," Diseases of the Colon and Rectum, vol. 57, no. 5, pp. 623-631, 2014.

[20] A. Jänes, Y. Cengiz, and L. A. Israelsson, "Randomized clinical trial of the use of a prosthetic mesh to prevent parastomal hernia," British Journal of Surgery, vol. 91, no. 3, pp. 280-282, 2004.

[21] A. Jänes, Y. Cengiz, and L. A. Israelsson, "Preventing parastomal hernia with a prosthetic mesh: a 5-year follow-up of a randomized study," World Journal of Surgery, vol. 33, no. 1, pp. 118-121, 2009.

[22] X. Serra-Aracil, J. Bombardo-Junca, J. Moreno-Matias et al., "Randomized, controlled, prospective trial of the use of a mesh to prevent parastomal hernia," Annals of Surgery, vol. 249, no. 4, pp. 583-587, 2009.

[23] M. López-Cano, R. Lozoya-Trujillo, S. Quiroga et al., "Use of a prosthetic mesh to prevent parastomal hernia during laparoscopic abdominoperineal resection: arandomized controlled trial," Hernia, vol. 16, no. 6, pp. 661-667, 2012.

[24] E. Târcoveanu, A. Vasilescu, E. Cotea et al., "Parastomal hernias-clinical study of therapeutic strategies," Chirurgia, vol. 109, no. 2, pp. 179-184, 2014.

[25] H. T. Brandsma, B. M. E. Hansson, T. J. Aufenacker et al., "Prophylactic mesh placement to prevent parastomal hernia, early results of a prospective multicentre randomized trial," Hernia, vol. 20, no. 4, pp. 535-541, 2016.

[26] J. R. Lambrecht, S. G. Larsen, O. Reiertsen, A. Vaktskjold, L. Julsrud, and K. Flatmark, "Prophylactic mesh at end-colostomy construction reduces parastomal hernia rate: a randomized trial," Colorectal Disease, vol. 17, no. 10, pp. O191-O197, 2015.

[27] M. Vierimaa, K. Klintrup, F. Biancari et al., "Prospective, randomized study on the use of a prosthetic mesh for prevention of parastomal hernia of permanent colostomy," Diseases of the Colon and Rectum, vol. 58, no. 10, pp. 943-949, 2015.

[28] M. López-Cano, X. Serra-Aracil, L. Mora et al., "Preventing parastomal hernia using a modified sugarbaker technique with composite mesh during laparoscopic abdominoperineal resection," Annals of Surgery, 2016.

[29] J. P. Higgins, D. G. Altman, P. C. Gotzsche et al., "The Cochrane Collaboration's tool for assessing risk of bias in randomised trials," British Medical Journal, vol. 343, Article ID d5928, 2011.
[30] L. Sun, R. Guo, and L. Sun, "Dexmedetomidine for preventing sevoflurane-related emergence agitation in children: a metaanalysis of randomized controlled trials," Acta Anaesthesiologica Scandinavica, vol. 58, no. 6, pp. 642-650, 2014.

[31] J. P. T. Higgins and S. G. Thompson, "Quantifying heterogeneity in a meta-analysis," Statistics in Medicine, vol. 21, no. 11, pp.15391558, 2002.

[32] A. Cingi, T. Cakir, A. Sever, and A. O. Aktan, "Enterostomy site hernias: a clinical and computerized tomographic evaluation," Diseases of the Colon and Rectum, vol. 49, no. 10, pp. 1559-1563, 2006.

[33] P. J. Arumugam, L. Bevan, L. Macdonald et al., "A prospective audit of stomas-analysis of risk factors and complications and their management," Colorectal Disease, vol. 5, no. 1, pp. 49-52, 2003.

[34] J. C. Goligher, O. V. Lloyd-davies, and C. T. Robertson, "Smallgut obstructions following combined excision of the rectum with special reference to strangulation round the colostomy," The British Journal of Surgery, vol. 38, no. 152, pp. 467-473, 1951.

[35] F. J. Burns, "Complications of colostomy," Diseases of the Colon and Rectum, vol. 13, no. 6, pp. 448-450, 1970.

[36] R. Kasperk, U. Klinge, and V. Schumpelick, “The repair of large parastomal hernias using a midline approach and a prosthetic mesh in the sublay position," American Journal of Surgery, vol. 179, no. 3, pp. 186-188, 2000.

[37] K. Marimuthu, C. Vijayasekar, D. Ghosh, and G. Mathew, "Prevention of parastomal hernia using preperitoneal mesh: a prospective observational study," Colorectal Disease, vol. 8, no. 8, pp. 672-675, 2006.

[38] I. Gögenur, J. Mortensen, T. Harvald, J. Rosenberg, and A. Fischer, "Prevention of parastomal hernia by placement of a polypropylene mesh at the primary operation," Diseases of the Colon and Rectum, vol. 49, no. 8, pp. 1131-1135, 2006.

[39] J. Ripoche, C. Basurko, P. Fabbro-Perray, and M. Prudhomme, "Parastomal hernia. A study of the French federation of ostomy patients," Journal of Visceral Surgery, vol. 148, no. 6, pp. e435e441, 2011.

[40] S. P. Wijeyekoon, K. Gurusamy, K. El-Gendy, and C. L. Chan, "Prevention of parastomal herniation with biologic/composite prosthetic mesh: a systematic review and meta-analysis of randomized controlled trials," Journal of the American College of Surgeons, vol. 211, no. 5, pp. 637-645, 2010.

[41] K.-W. Tam, P.-L. Wei, L.-J. Kuo, and C.-H. Wu, "Systematic review of the use of a mesh to prevent parastomal hernia," World Journal of Surgery, vol. 34, no. 11, pp. 2723-2729, 2010.

[42] J. Shabbir, B. N. Chaudhary, and R. Dawson, "A systematic review on the use of prophylactic mesh during primary stoma formation to prevent parastomal hernia formation," Colorectal Disease, vol. 14, no. 8, pp. 931-936, 2012.

[43] L. A. Israelsson, "Preventing and treating parastomal hernia," World Journal of Surgery, vol. 29, no. 8, pp. 1086-1089, 2005.

[44] A. R. Janson, A. Jänes, and L. A. Israelsson, "Laparoscopic stoma formation with a prophylactic prosthetic mesh," Hernia, vol. 14, no. 5, pp. 495-498, 2010.

[45] A. Hotouras, J. Murphy, M. Thaha, and C. L. Chan, "The persistent challenge of parastomal herniation: a review of the literature and future developments," Colorectal Disease, vol. 15, no. 5, pp. e202-e214, 2013.

[46] G. El-Gazzaz, R. P. Kiran, and I. Lavery, "Wound complications in rectal cancer patients undergoing primary closure of the perineal wound after abdominoperineal resection," Diseases of the Colon and Rectum, vol. 52, no. 12, pp. 1962-1966, 2009. 
[47] A. Biswas, K. Marimuthu, and G. Mathew, "Prevention of parastomal hernia using pre-peritoneal mesh-long term outcome of a prospective study," Acta Chirurgica Belgica, vol. 115, no. 1, pp. 15-19, 2015.

[48] L. Lee, A. Saleem, T. Landry, E. Latimer, P. Chaudhury, and L. S. Feldman, "Cost effectiveness of mesh prophylaxis to prevent parastomal hernia in patients undergoing permanent colostomy for rectal cancer," Journal of the American College of Surgeons, vol. 218, no. 1, pp. 82-91, 2014.

[49] M. López-Cano and X. Serra-Aracil, "Parastomal hernia prevention through laparoscopic modified Sugarbaker technique with composite mesh (Physiomesh ${ }^{\circledR}$ )," Cirugia Espanola, vol. 91, no. 5, pp. 331-334, 2013.

[50] M. Skrovina, J. Bartoš, E. Holášková, M. Migrová, K. Klos, and P. Anděl, "Laparoscopic parastomal hernia repair with modified Sugarbaker technique in patients after abdominoperineal resection of the rectum-initial experience," Rozhledy v Chirurgii, vol. 93, no. 10, pp. 502-506, 2014.

[51] B. M. E. Hansson, S. Morales-Conde, T. Mussack, J. Valdes, F. E. Muysoms, and R. P. Bleichrodt, "The laparoscopic modified Sugarbaker technique is safe and has a low recurrence rate: a multicenter cohort study," Surgical Endoscopy, vol. 27, no. 2, pp. 494-500, 2013.

[52] P. Hauters, J.-L. Cardin, M. Lepere et al., "Long-term assessment of parastomal hernia prevention by intra-peritoneal mesh reinforcement according to the modified Sugarbaker technique," Surgical Endoscopy, 2016.

[53] E. E. Londono-Schimmer, A. P. K. Leong, and R. K. S. Phillips, "Life Table analysis of stomal complications following colostomy," Diseases of the Colon \& Rectum, vol. 37, no. 9, pp. 916-920, 1994. 


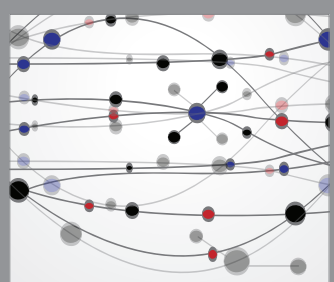

The Scientific World Journal
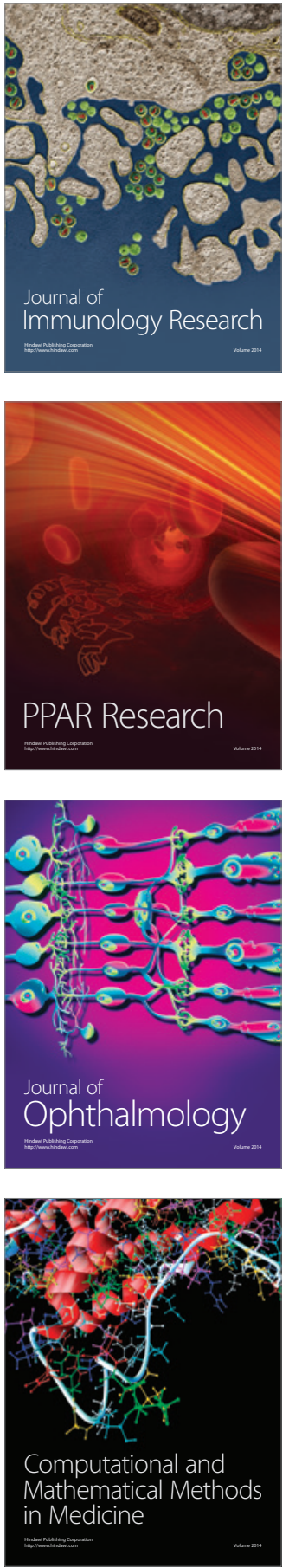

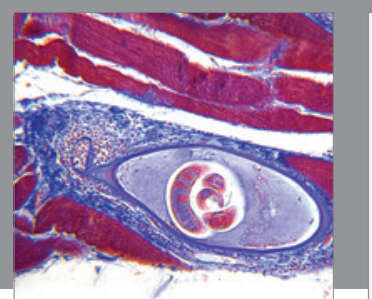

Gastroenterology Research and Practice

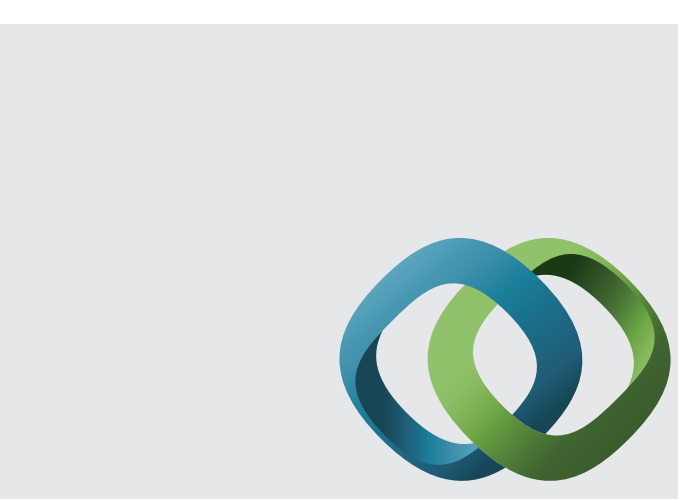

\section{Hindawi}

Submit your manuscripts at

http://www.hindawi.com
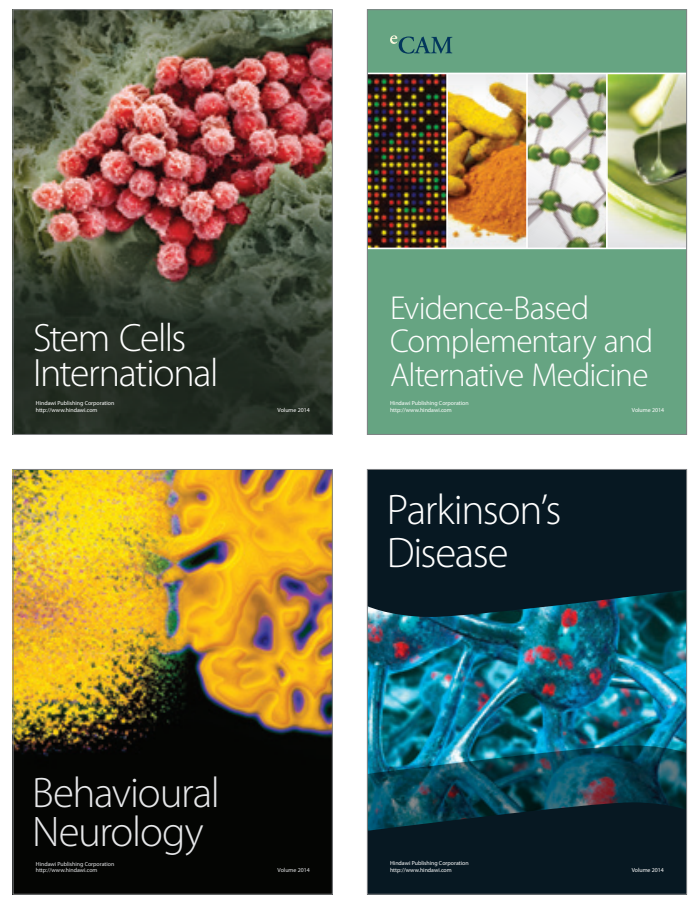
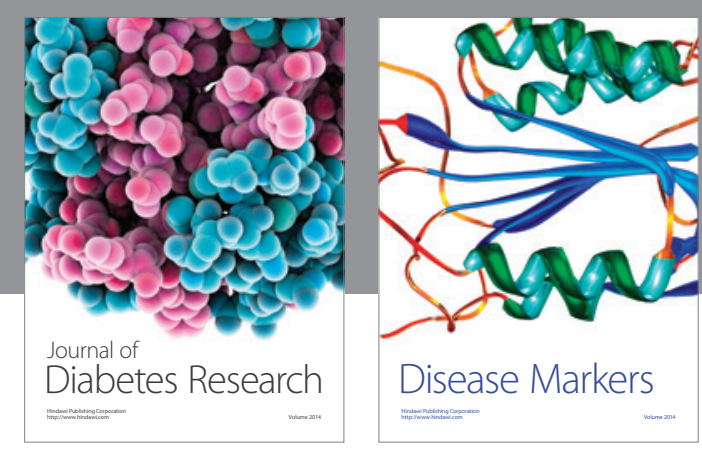

Disease Markers
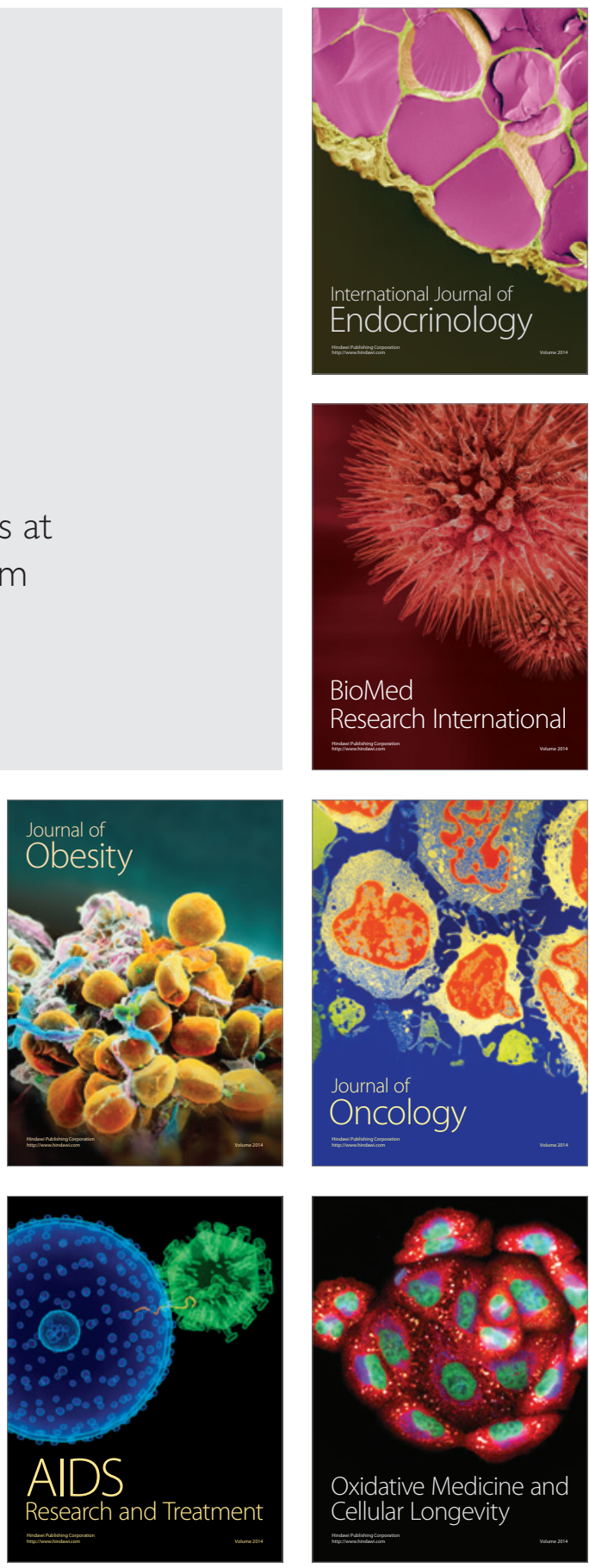